\title{
Effect of automation on prothrombin time test in NEQAS surveys
}

\author{
L POLLER, J M THOMSON, D A TABERNER The UK Reference Laboratory for Anticoagulant \\ Reagents and Control, Withington Hospital, Manchester
}

SUMMARY The performance of coagulometers in the National Quality Assessment Scheme (NEQAS) surveys of the prothrombin time conducted between 1986 and 1987 was reviewed. There were sufficient data for analysis for eight types of coagulometer used with a single type of thromboplastin reagent and one instrument with an alternative reagent. The overall reliability efficacy of each instrument was evaluated by determining the orthogonal regression slope parameters for prothrombin time (PT) and international normalised ratios (INR) using the manual technique as the reference method. Seven of the eight types of coagulometer tended to overestimate the INR. A pattern frequently observed with coagulometers, and difficult to regulate, was a trend to underestimate INR below 3.0 and overestimate higher INR. Overestimation of INR values over 3.0 was particularly pronounced with three types of instrument (Fibrintimer, Lancer, KC4/10). The KC4/10 was used by a sufficient number of participants to permit analysis of the performance of individual instruments. Within instrument differences were similar to those produced by different types of coagulometers. Thromboplastin reagents affected the INR values obtained with coagulometers.

The study indicates that each local reagent-instrument combination must be calibrated by the participant to obtain reliable INR values. The use of a general correction factor for a local PT system seems to be invalid owing to the considerable variation in performance of individual coagulometers. The two best guides to the choice of coagulometer may be the deviation from the manual result and precision estimated by the coefficient of variation of the INR.

The number of centres using automated and semiautomated techniques for the prothrombin time (PT) is steadily increasing in the United Kingdom. According to National External Quality Assessment Scheme (NEQAS) survey returns, by 1988 about $50 \%$ of hospitals were using a coagulometer. There have been many changes in procedures since our previous report on automated instruments.' We have therefore limited the analysis to the results of the last two years' NEQAS surveys. This period was chosen because in 1986 there was a major change in methodology in the United Kingdom with the withdrawal of the Manchester Comparative Reagent human brain thromboplastin, and the spectrum of instruments is constantly changing.

\section{Material and methods}

Results of the serial NEQAS surveys of the PT between April 1986 and October 1987 were reviewed. These provided a total of 20 test plasmas for inclusion

Accepted for publication 4 August 1988 in the study. All samples were from patients receiving anticoagulant treatment with nicoumalone (Sinthrome) and were obtained from single donors by plasmapheresis.

The number of unsatisfactory performers in NEQAS (greater than $15 \%$ deviation from the reagent manual mean) for four surveys up to January 1988 was reviewed according to whether a manual or an automated technique was used.

The mean PT and INR obtained by the manual technique, which is the traditional reference method, were compared with the mean results obtained with the various automated and semiautomated methods. One brand of thromboplastin ISI $1 \cdot 1$ (reagent A) was used by most of the centres and provided sufficient results for analysis with the following eight instruments: Biodata, Biomatic 2000, Burkard S \& G, Coag-a-Pet (dual channel), Coag-a-Mate $\times 2$, Fibrintimer, $\mathrm{KC} 4 / \mathrm{KC} 10$ and Lancer. An instrument was included only if there was a minimum of four users in any single survey and the average over the series was at least six. This is less than the customary minimum regarded as sufficient for separate analysis in NEQAS, 
but it was thought that the summation of data from the series of exercises would provide a sufficient number of results for meaningful comparisons to be made. A second thromboplastin (reagent B) ISI 1.4 was used in sufficient numbers for analysis with only one instrument type $(\mathrm{KC} / \mathrm{KC10})$ but provided comparative data on the reagent-instrument interaction with that system.

The mean coagulometer PT for each survey plasma was plotted using orthogonal regression analysis against the mean manual result with the same plasma on a logarithmic scale. The slope parameter and its coefficient of variation (CV) were determined. The same analysis was then repeated using the INR instead of the PT values. Results of PTs and INR were analysed separately to observe the effects of automation on the two different methods of reporting and to determine whether the INR system of PT standardisation would compensate for any possible instrument effects. The INR results reported by participants for the 20 plasmas were analysed according to the combination of coagulometer and reagent and compared with the appropriate reagent manual mean. As an assessment of the precision of the systems, results were expressed as an overall mean INR with the mean CV.

Finally, the within instrument variability using the same type of coagulometer was studied with the $\mathrm{KC} 4 / 10$ system as this was used by a far larger number of participants than any other instrument. The results of PT and INR of each individual $\mathrm{KC} 4 / \mathrm{KCl}$ coagulometer were plotted using the same set of NEQAS test plasmas against the overall manual mean on a logarithmic scale. This was to assess the degree of variation in slope between users of this instrument. Results from 15 centres using reagent $A$, and nine using reagent $\mathbf{B}$, were compared.

\section{Results}

The percentage of unsatisfactory performers in the most recent surveys was seen to be higher with automation and was also influenced by the thromboplastin (table 1).

The estimated orthogonal regression slope parameters for PT and INR with the different systems are given in table 2, together with the numbers of users in the serial surveys. The slopes of the mean data for all centres with the two methods of reporting were almost identical. Seven of the mean slopes for the eight instrument types were less than $1 \cdot 0$, the theoretical figure for the slope with the manual technique, and ranged from 0.91 to 0.97 . The precision $(\mathrm{CV} \%)$ of the estimation of the slope also varied between the types of coagulometer and the two methods of reporting. The $\mathrm{CV}$ of the slope for the INR tended to be greater than the slope for the PT.

Table 2 indicates that with seven of the instruments there was an overall trend to overestimate the INR-
Table 1 Percentage of unsatisfactory performers in three most recent surveys (57-59) included in analysis

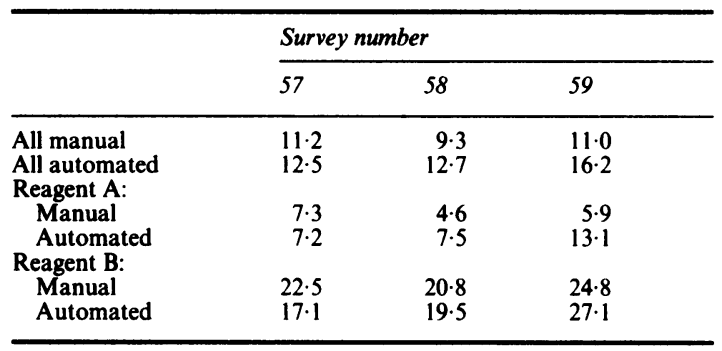

Table 2 Overall mean regression slopes and CV

\begin{tabular}{llll}
\hline & \multicolumn{2}{l}{ Estimated slope parameter } \\
\cline { 2 - 3 } Instrument & $P T(C V)$ & $I N R(C V)$ & $\begin{array}{l}\text { Average no } \\
\text { of users }\end{array}$ \\
\hline Reagent A & & & \\
Coag-a-Mate $\times 2$ & $0.97(1.98)$ & $0.97(1.89)$ & 17 \\
Fibrintimer & $0.93(1.27)$ & $0.92(1.62)$ & 15 \\
Biodata & $0.95(1.69)$ & $0.94(1.94)$ & 7 \\
Lancer & $0.91(2.38)$ & $0.89(2.93)$ & 6 \\
Coag D/C & $0.95(2.58)$ & $0.95(2.57)$ & 6 \\
Burkard & $1.02(1.63)$ & $1.01(2.43)$ & 16 \\
Bio 2000 & $0.94(1.92)$ & $0.92(2.49)$ & 8 \\
KC4/KC10 & $0.93(1.42)$ & $0.92(1.60)$ & 24 \\
Reagent B & & & \\
KC4/10 & $0.86(2.0)$ & $0.86(1.67)$ & 9 \\
\hline
\end{tabular}

that is slope $<1.0$-but with three of these (Fibrintimer, Lancer and KC4/10) this tendency was only evident with high INR values. With these three instruments there was a transition from underestimation to overestimation which seemed to take place at the mid therapeutic zone-that is with an INR of about 3.0. This effect was also observed with about two thirds of the $\mathrm{KC} 4 / 10$ users when results from individual laboratories were examined. An example of a centre showing this effect is seen in fig 1 . Another laboratory with the same type of instrument gave a more satisfactory correlation (fig 2).

When the effect of a reagent-instrument interaction on $\log$ PTs was studied for the KC4/KC10 system, a different slope was observed with the two rabbit thromboplastins-that is, 0.93 for reagent $A$ and 0.86 for reagent $B$. Tables 3 and 4 give the orthogonal regression slopes obtained at individual centres using the $\mathrm{KC4} / 10$ with reagents $A$ and $B$, respectively, showing the range of variation of the slope obtained from the data of the respective laboratories. The slopes of the INR values for individual laboratories with the $\mathrm{KC} 4 / 10$ ranged between 0.80 and 0.96 for reagent $\mathrm{A}$; mean INR value slopes for the whole group of different coagulometers used with reagent $A$ was 0.89 1.01 . The within instrument differences were similar to those produced by different coagulometers.

The INR results with coagulometers reported by participants were compared with the corresponding manual mean obtained in the NEQAS surveys (table 5). Although the mean results compared well with all 


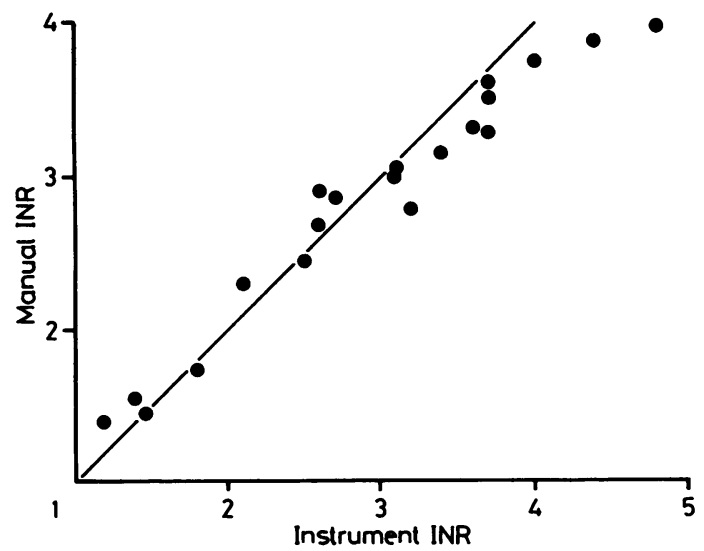

Fig 1 Instrument with non-linear relation.

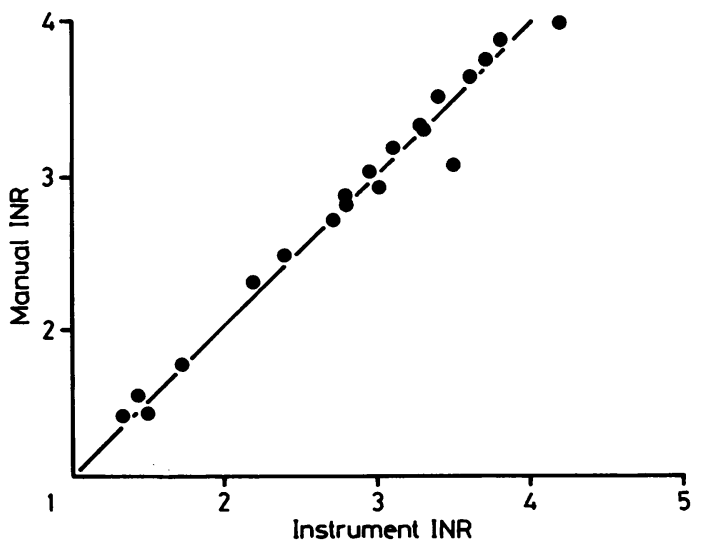

Fig 2 A more linear correlation than that seen in fig 1.

Figs 1 and 2 INR obtained by two centres using KC/10 instruments correlated with respective overall reagent manual mean INR.

coagulometers, there were obvious coagulometer effects, with the mean INR ranging from 2.67 to 2.93 for reagent $A$. The precision estimated by the $C V$ of the INR varied between 6.93 and 12.88 for this reagent. Seven of the eight systems gave less precision than the manual method. In contrast with reagent $B$, the manual technique gave the largest recorded $C V$ of the INR. The one instrument used in sufficient numbers with this reagent, the $\mathrm{KC} 4 / 10$, improved this result.

\section{Discussion}

Over the past decade an increasing number of United Kingdom laboratories have adopted automated and semiautomated methods for PT testing. By late 1987 almost half the hospitals were using an automated

Table 3 Regression slopes of manual result against automated (KC/10) with Reagent $A$

\begin{tabular}{llll} 
& \multicolumn{2}{l}{ Slope } & \\
\cline { 2 - 3 } Laboratory & $P T$ & INR & No of plasmas \\
\hline 1 & 0.86 & 0.83 & 17 \\
2 & 0.84 & 0.88 & 16 \\
3 & 0.85 & 0.80 & 19 \\
4 & 0.89 & 0.85 & 16 \\
5 & 0.94 & 0.89 & 19 \\
6 & 0.86 & 0.83 & 18 \\
7 & 0.88 & 0.88 & 20 \\
8 & 0.93 & 0.96 & 20 \\
9 & 0.87 & 0.89 & 16 \\
10 & 0.91 & 0.89 & 17 \\
11 & 0.94 & 0.95 & 17 \\
12 & 0.87 & 0.83 & 20 \\
13 & 0.87 & 0.86 & 20 \\
14 & 0.91 & 0.87 & 19 \\
15 & 0.97 & 0.94 & 17 \\
Mean & 0.89 & 0.88 & \\
SD & 0.04 & 0.05 & \\
Range & $0.84-0.97$ & $0.80-0.96$ & \\
\hline
\end{tabular}

Table 4 Regression slopes of manual result against automated $(K C / 10)$ with Reagent $B$

\begin{tabular}{llll}
\hline & \multicolumn{2}{l}{ Slope } & \\
\cline { 2 - 3 } Laboratory & $P T$ & INR & No of plasmas \\
\hline 16 & 0.80 & 0.82 & 19 \\
17 & 0.84 & 0.82 & 20 \\
18 & 0.89 & 0.85 & 16 \\
19 & 0.83 & 0.89 & 20 \\
20 & 0.89 & 0.92 & 20 \\
21 & 0.89 & 0.81 & 16 \\
22 & 0.85 & 0.85 & 19 \\
23 & 0.86 & 0.84 & 20 \\
24 & 0.89 & 0.87 & 17 \\
Mean & 0.86 & 0.85 & \\
SD & 0.03 & 0.04 & \\
Range & $0.80-0.89$ & $0.81-0.92$ & \\
\hline
\end{tabular}

procedure for routine work. A variety of instruments based on different principles of end point detection are used.

Serial NEQAS surveys have indicated that there is

Table 5 Comparison of mean INR results with various coagulometers

\begin{tabular}{lll}
\hline & \multicolumn{2}{l}{ Reagent $A$} \\
\cline { 2 - 3 } Instrument & Mean INR & Mean CV\% \\
\hline Coag-a-mate $\times 2$ & $2 \cdot 78$ & $10 \cdot 12$ \\
Fibrintimer & 2.93 & $8 \cdot 57$ \\
Biodata & $2 \cdot 75$ & $6 \cdot 93$ \\
Lancer & 2.97 & $9 \cdot 78$ \\
Coag DC & $2 \cdot 67$ & $9 \cdot 30$ \\
Burkard & $2 \cdot 73$ & $10 \cdot 38$ \\
Bio 2000 & $2 \cdot 88$ & $12 \cdot 88$ \\
KC4/10 & $2 \cdot 88$ & 8.96 \\
Manual & $2 \cdot 85$ & $8 \cdot 62$ \\
& & \\
KC4/10 & Reagent B & 10.33 \\
Manual & 2.93 & 14.09 \\
\hline
\end{tabular}


considerable disagreement between the mean INR values obtained on the test plasmas with the various automated methods, although some give good agreement with the manual result. The latter is regarded in the United Kingdom as the reference value and used to define poor performance. This may have produced a degree of bias in increasing the numbers of unsatisfactory performances with coagulometers as performance in NEQAS is judged against the manual mean. The impression remains, however, that overall standards of performance have not been improved by the more widespread adoption of automation in the United Kingdom.

Data from NEQAS surveys had suggested that coagulometers had an individual effect on the INR values. The effect varied according to the routine thromboplastin used. Only one type of instrument, the $\mathrm{KC} 4 / \mathrm{KCl} 10$, was used in sufficient numbers for analysis with more than one reagent. The different slopes, 0.93 and 0.86 , respectively, for reagents $A$ and B confirmed that pooling the data of coagulometer performance over the range of thromboplastins is therefore invalid. This agrees with the published data from the College of American Pathologists' surveys ${ }^{2}$ and serial reports from the United Kingdom NEQAS surveys. It was therefore considered advisable to restrict the analysis to users of one reagent used by about $75 \%$ of United Kingdom centres. To permit reasonable statistical analysis in the present survey the observations were limited to the effects of the eight instruments used by an average of six or more participants with this reagent. Of these, however, only four, KC4/10, Burkard, Coag-a-mate $\times 2$ and Fibrintimer were used by 15 or more laboratories in each survey and therefore more confidence can be placed in their mean results.

Although they differed across the range of instruments, the regression slopes were similar, irrespective of whether they are analysed as PTs or INR. With six of the eight instruments evaluated, the CV of the slope of the INR was greater than the CV of the slope of the PT. This implies that there was an additional error in the conversion of the PTs to INR, and the international sensitivity index (ISI)/INR system was of no assistance in reducing the coagulometer effects.

With reagent $A$, the mean slopes showed a degree of variation and they were significantly different from the manual mean (1.0 by definition). With seven of the eight coagulometers the value was less than unity and it therefore would be expected that all their INR results would be greater than the manual INR results. In practice, the effect proved variable across the range of INR values represented in the NEQAS survey samples. With some instruments the mean manual and automated INR values tend to approximate to one another towards the middle of the 2.0-4.5 INR range but the coagulometer INRs were lower at less intense and higher at more intense degrees of anticoagulation.
Therefore, the value for the slope with each coagulometer (table 2) could not be used to predict or correct for the instrument variable. The $\mathrm{CV}$ of the slopes provides a guide to the precision of coagulometers. This varied considerably with different types of instrument.

An interesting and unexpected finding was derived from examining the performance of individual instruments of the same manufacturer and type (KC4/ $\mathrm{KC10}$ ). This particular instrument performed well in NEQAS surveys and there is no reason to believe, therefore, that the individual variation among users would be greater than the other seven listed. Its CV was lower than the manual technique both with PTs and INR. Fifteen users of this system with reagent $A$ and nine with reagent $B$ tested at least 16 of the 20 plasmas. Their slope values showed more variation with reagent A (0.84 to $0.97 \mathrm{PT}, 0.80$ to $0.96 \mathrm{INR})$ than the mean slopes across the range of instruments $(0.91$ to $1.02 \mathrm{PT}, 0.89$ to $1.01 \mathrm{INR}$ ). There were similar findings with reagent $B$. This indicates that the local variable with each $\mathrm{KC} 4 / 10$ coagulometer is at least as great as the differences introduced by the range of instruments systems. With some $\mathrm{KC} 4 / 10$ instruments there was a noticeable tendency to overestimate high INR but this was not uniform as some showed a more linear response. The observation suggests that there is a need for adjustment of individual instruments to obtain linearity of INR values. Each coagulometer must be calibrated locally by participants as the use of a correction factor, or adjustment of the ISI for all models of one instrument type, seems to be invalid. A range of plasmas calibrated in INR might assist users of instruments to either correct for the local instrument variable or permit adjustment of the coagulometer to achieve the desired result.

Perhaps the two best rapid guides to the reliability of a coagulometer are its deviation from the manual results and its precision estimated by the $\mathrm{CV}$ of the INR for the particular instrument group. Table 5 provides these data and shows that instruments with the best precision do not necessarily give the result which approximates best to the manual method. The choice with the present generation of coagulometers may therefore be a compromise between these parameters.

\section{References}

1 Poller L, Thomson Jean M, Yee KF. Automated versus manual techniques for the prothrombin time test: results of proficiency assessment studies. Br J Haematol 1977;38:391-9.

2 Evatt BL, Brogan D, Triplett DA, Koepke JA, Waters G. Influences of thromboplastin and instrumentation upon prothrombin time tests: implications for standardisation. In: Triplett DA, ed. Standardisation of coagulation assays: an overview. Skokie, Illinois: College of American Pathologists, 1982:39-47.

Requests for reprints to: Dr L Poller, Director, UK Reference Laboratory for Anticoagulant Reagents and Control, Withington Hospital, Manchester M20 8LR, England. 